\title{
A New DBMS Architecture for DB-IR Integration
}

\author{
Kyu-Young Whang \\ Computer Science Department and \\ Advanced Information Technology Research Center(AITrc) \\ KAIST, Korea \\ kywhang@cs.kaist.ac.kr
}

\begin{abstract}
Nowadays, as there is an increasing need to integrate the DBMS (for structured data) with Information Retrieval (IR) features (for unstructured data), DB-IR integration becomes one of major challenges in the database area 12. Extensible architectures provided by commercial ORDBMS vendors can be used for DB-IR integration. Here, extensions are implemented using a high-level (typically, SQL-level) interface. We call this architecture loose-coupling. The advantage of loose-coupling is that it is easy to implement. But, it is not preferable for implementing new data types and operations in large databases when high performance is required. In this talk, we present a new DBMS architecture applicable to DB-IR integration, which we call tight-coupling. In tight-coupling, new data types and operations are integrated into the core of the DBMS engine in the extensible type layer. Thus, they are incorporated as the "first-class citizens" [1] within the DBMS architecture and are supported in a consistent manner with high performance. This tight-coupling architecture is being used to incorporate IR features and spatial database features into the Odysseus ORDBMS that has been under development at KAIST/AITrc for over 16 years 3. In this talk, we introduce Odysseus and explain its tightly-coupled IR features (U.S. patented in 2002 2]). Then, we demonstrate excellence of tight-coupling by showing benchmark results. We have built a web search engine that is capable of managing 20 100 million web pages in a non-parallel configuration using Odysseus. This engine has been successfully tested in many commercial environments. In a parallel configuration, it is capable of managing billons of web pages. This work won the Best Demonstration Award from the IEEE ICDE conference held in Tokyo, Japan in April 2005 3 .
\end{abstract}

\section{About the Speaker}

Kyu-Young Whang is Professor of Computer Science and Director of Advanced Information Technology Research Center (AITrc) at KAIST. Previously, he was with IBM T.J.Watson Research Center from 1983 to 1990. Since joining KAIST in 1990, he has been leading the Odysseus DBMS project featuring tight-coupling of DBMS with information retrieval (IR) and spatial functions. Dr. Whang is one of the pioneers of probabilistic counting, which nowadays is being widely used in 
approximate query answering, sampling, and data streaming. One of the algorithms he co-developed at IBM Almaden (then San Jose) Research Lab in 1981 has been made part of DB2. Dr. Whang is the author of the first main-memory relational query optimization model developed in 1985 and reported in 1990 in ACM TODS in the context of Office-by-Example (OBE). This model influenced subsequent optimization models of commercial main-memory DBMSs. His research has covered a wide range of database issues including physical database design, query optimization, DBMS engine technologies, and more recently, IR, spatial databases, data mining, and XML. Dr. Whang is a Co-Editor-in-Chief of the VLDB Journal, having served the journal for 17 years from its inception as its founding editorial board member. He is a Trustee Emeritus of the VLDB Endowment and served the international academic community as the General Chair of VLDB2006, DASFAA2004, and PAKDD2003, as a PC Co-Chair of VLDB2000, CoopIS1998, and ICDE2006, and as an editorial board member of journals such as IEEE TKDE and IEEE Data Engineering Bulletin. He served as an IEEE Distinguished Visitor from 1989 to 1990. He earned his Ph.D. from Stanford University in 1984. Dr. Whang is an IEEE Fellow, a member of the ACM and IFIP WG 2.6.

\section{References}

1. Abiteboul, S. et al., "The Lowell Database Research Self-Assessment," Communications of the ACM, Vol.48, No.5, pp. 111-118, May 2005.

2. Whang, K., Park, B., Han, W., and Lee, Y., "An Inverted Index Storage Structure Using Subindexes and Large Objects for Tight Coupling of Information Retrieval with Database Management Systems," U.S. Patent No. 6,349,308, Feb. 19, 2002 (Appl. No. 09/250,487, Feb. 15, 1999).

3. Whang, K., Lee, M., Lee, J., Kim, M., and Han, W., "Odysseus: a High-Performance ORDBMS Tightly-Coupled with IR Features," In Proc. IEEE 21st Int'l Conf. on Data Engineering (ICDE), Tokyo, Japan, Apr. 5-8, 2005. This paper received the Best Demonstration Award. 